\title{
Perfil de mães de recém-nascidos pré-termo em um município do semiárido cearense
}

\author{
Profile of pre-term newborn mothers in a municipality in the cearense semiarid \\ Perfil de madres recién nacidas prematuras en un municipio de la semiárida de cearense
}

Recebido: 08/02/2021 | Revisado: 12/02/2021 | Aceito: 25/02/2021 | Publicado: 03/03/2021

\author{
Maria Danara Alves Otaviano \\ ORCID: https://orcid.org/0000-0003-1166-7428 \\ Universidade Federal do Ceará, Brasil \\ E-mail: otaviano23danara@gmail.com \\ Maria Adelane Monteiro da Silva \\ ORCID: https://orcid.org/0000-0001-7579-2645 \\ Universidade Estadual Vale do Acaraú, Brasil \\ E-mail: adelanemonteiro@hotmail.com \\ Francisca Júlia dos Santos Sousa \\ ORCID: https://orcid.org/0000-0002-7883-9725 \\ Universidade Federal do Ceará, Brasil \\ E-mail: juliasantossousa@ hotmail.com \\ Anna Larissa Moraes Mesquita \\ ORCID: https://orcid.org/0000-0002-7429-5214 \\ Universidade Federal do Ceará, Brasil \\ E-mail: larissamoraesmesquita@gmail.com \\ Tainá de Jesus Alves Portela \\ ORCID: https://orcid.org/0000-0003-1422-3240 \\ Universidade Estadual Vale do Acaraú, Brasil \\ E-mail: tainadejesusalves@gmail.com \\ Maria Girlane Sousa Albuquerque Brandão \\ ORCID: https://orcid.org/0000-0002-9925-4750 \\ Universidade Estadual Vale do Acaraú, Brasil \\ E-mail: girlane.albuquerque@yahoo.com.br \\ Francisca Erandielly Lopes Sousa \\ ORCID: https://orcid.org/0000-0003-0313-4840 \\ Universidade Estadual Vale do Acaraú, Brasil \\ E-mail: erandiellysousa@hotmail.com
}

\begin{abstract}
Resumo
Objetivo: Delinear o perfil socioeconômico e obstétrico de mães com filhos pré-termo em um município do semiárido cearense. Métodos: Trata-se de um estudo descritivo, documental, retrospectivo, com abordagem quantitativa. A fonte utilizada para a coleta foi a base de dados de um município da região norte do Estado do Ceará, alimentada a partir de entrevistas aplicadas a todas as puérperas que deram à luz nas maternidades locais no ano de 2015. As informações foram processadas usando o software EPI INFO 6.0 e o programa Excel 2007. Os dados foram analisados por meio de estatística descritiva. Os princípios da Resolução $\mathrm{n}^{\circ}$ 466/2012, aprovados pelo Comitê de Ética sob número de protocolo 1.590.501, foram respeitados. Resultados: O perfil socioeconômico das puérperas no ano de 2015 foi de $74,6 \%$ na faixa etária de 20 a 35 anos e $45,7 \%$ com renda menor que um salário-mínimo 37,8\% com ensino médio completo. Quanto ao perfil obstétrico, $88,1 \%$ tinha de 1 a 3 gestações e $51,1 \%$ realizou sete ou mais consultas de prénatal. O tipo de parto prevalente foi à cesárea $(69,8 \%)$. As intercorrências evidenciadas foram: infecção urinária, ameaça de parto prematuro e infecção vaginal, associada a parto prematuro. As puérperas de menor renda apresentaram maiores porcentagens de infecção do trato urinário e infecção vaginal. Conclusões: As características encontradas em mulheres com parto prematuro neste estudo não diferem dos achados da literatura, contudo, evidenciou-se a relação da infecção urinária com a prematuridade e a influência das condições socioeconômicas neste desfecho neonatal.
\end{abstract}

Palavras-chave: Recém-nascido prematuro; Fatores de risco; Saúde da mulher.

\begin{abstract}
Objective: To outline the socioeconomic and obstetric profile of mothers with preterm children in a municipality in the semi-arid region of Ceará. Methods: This is a descriptive, documentary, retrospective study, with a quantitative approach. The source used for the collection was the database of a municipality in the northern region of the State of Ceará, fed from interviews applied to all mothers who gave birth in local maternity hospitals in 2015. The information was processed using the EPI INFO 6.0 software and Excel 2007 program. Data were analyzed using descriptive statistics. The principles of Resolution 466/2012, approved by the Ethics Committee under protocol number
\end{abstract}


1.590.501, were respected. Results: The socioeconomic profile of the puerperal women in 2015 was $74.6 \%$ in the age group of 20 to 35 years old and $45.7 \%$ with an income below the minimum wage $37.8 \%$ with complete high school education. As for the obstetric profile, $88.1 \%$ had 1 to 3 pregnancies and $51.1 \%$ had seven or more prenatal consultations. The prevalent type of delivery was cesarean $(69.8 \%)$. The complications observed were: urinary tract infection, threat of premature birth and vaginal infection, associated with premature birth. Low-income mothers had higher percentages of urinary tract infection and vaginal infection. Conclusions: The characteristics found in women with premature birth in this study do not differ from the findings in the literature, however, the relationship between urinary infection and prematurity and the influence of socioeconomic conditions on this neonatal outcome was evident.

Keywords: Infant premature; Risk factors; Women's health.

\section{Resumen}

Objetivo: Esbozar el perfil socioeconómico y obstétrico de madres con niños prematuros en un municipio de la región semiárida de Ceará. Métodos: Se trata de un estudio descriptivo, documental, retrospectivo, con enfoque cuantitativo. La fuente utilizada para la recolección fue la base de datos de un municipio de la región norte del estado de Ceará, alimentada a partir de entrevistas aplicadas a todas las madres que dieron a luz en maternidades locales en 2015. La información fue procesada mediante el software EPI INFO 6.0 y Programa Excel 2007. Los datos se analizaron mediante estadística descriptiva. Se respetaron los principios de la Resolución 466/2012, aprobada por el Comité de Ética con el número de protocolo 1.590.501. Resultados: El perfil socioeconómico de las puérperas en 2015 fue de $74,6 \%$ en el grupo de edad de 20 a 35 años y de 45,7\% con ingresos inferiores al salario mínimo 37,8\% con estudios secundarios completos. En cuanto al perfil obstétrico, el 88,1\% tuvo de 1 a 3 embarazos y el 51,1\% tuvo siete o más consultas prenatales. El tipo de parto predominante fue la cesárea $(69,8 \%)$. Las complicaciones observadas fueron: infección del tracto urinario, amenaza de parto prematuro e infección vaginal, asociada a parto prematuro. Las madres de bajos ingresos tenían porcentajes más altos de infección del tracto urinario e infección vaginal. Conclusiones: Las características encontradas en mujeres con parto prematuro en este estudio no difieren de los hallazgos en la literatura, sin embargo, la relación entre infección urinaria y prematurez y la influencia de las condiciones socioeconómicas en este resultado neonatal fue evidente.

Palabras clave: Recien nacido prematuro; Factores de riesgo; Salud de la mujer.

\section{Introdução}

O termo prematuridade é definido segundo a Organização Mundial de Saúde (OMS), como o nascimento que ocorre antes das 37 semanas de gestação podendo ser classificados em: limítrofes $\left(35^{\mathrm{a}}-<37^{\mathrm{a}}\right.$ semana $)$, intermediários $\left(32^{\mathrm{a}}-<35^{\mathrm{a}}\right.$ semana), muito prematuros $\left(28^{\mathrm{a}}-<32^{\mathrm{a}}\right.$ semana) e extremos $\left(<28^{\mathrm{a}}\right.$ semana) (Hackbarthet et al., 2015).

A prematuridade configura-se em uma prioridade de Saúde Pública, uma vez que trata-se da causa mais importante de morte neonatal e a segunda causa principal de mortalidade em crianças menores de 5 anos. No relatório da OMS o Brasil está entre as 10 nações com maior número de nascimentos pré-termo, com taxas próximas as dos Estados Unidos da América, Índia e Nigéria demonstrando-se tratar de um problema global. A Pesquisa Nascer no Brasil identificou uma prevalência de 11,3\% de prematuridade no país. Já em estudo de correção de prevalência de nascimentos pré-termos realizado em Minas Gerais, as estimativas foram de 11,7 a 11,8\% no triênio 2009-2011 (Guimarães et al., 2017).

Ao analisar a evolução da prematuridade a partir dos dados do Sistema de Informação de Nascidos Vivos (SINASC), o Nordeste apresentou para os anos de 2000 e 2010, percentuais de 6,2\% e 5,9\%, respectivamente (Gonzaga et al., 2016). O estado sucedeu um pico na taxa de mortalidade neonatal (morte de crianças menores de 28 dias) no ano de 2016, atingindo 17,60. Ressalta-se que foram registrados elevados percentuais de óbitos neonatais precoces, a linha guia Nascer do Ceará (2017) destaca a inversão do estado em seus componentes da mortalidade infantil, em que os óbitos pós natais (morte de crianças maiores de 27 dias) apresentam uma redução e os óbitos neonatais representam quase a totalidade, somando $70,1 \%$ dessas mortes ainda no ano de 2016.

Em um hospital do Ceará, a taxa de prematuros extremos correspondeu a 5,2\% dos partos pré-termo, índice considerado elevado para o estado, em comparação a outros estudos similares, em que o trabalho de parto prematuro extremo 
foi de 1 a $2 \%$ dos nascimentos; identificou-se ainda que 73,9\% das mulheres realizou um quantitativo de 03 consultas, em discordância ao mínimo de 06 preconizadas pelo Programa de Humanização no Pré-natal e Nascimento (Ferreira et al., 2018).

Outro estudo também realizado na região verificou que 78,94\% dos óbitos neonatais na primeira semana de vida foram de recém-nascidos pré-termo, destaca-se que metade dos recém-nascidos prematuros nasceram com algum problema de saúde em comparação a praticamente todos os bebês a termo nascidos no mesmo período, que tiveram um nascimento saudável (Teixeira et al., 2018).

Destaca-se que no município em estudo, um dos três com os melhores resultados em relação às políticas sociais ofertadas na região semiárida, conforme o Sistema de Informações sobre Nascidos Vivos em 2016 a taxa de prematuridade foi de 14,38\%, em 2015 de 14,31\% e em 2018 de 13,23\% evidenciando-se uma leve regressão, contudo, ainda sendo vista como elevadas quando comparadas ao nordeste, que durante o mesmo período teve como maior taxa de prematuridade um percentual de $11,11 \%$ no ano de 2016.

Embora a etiologia da prematuridade seja multifatorial, a predisposição ao parto prematuro é associada a alguns fatores. Dentre eles, são estudados os aspectos comportamentais e sociodemográficos, as condições médicas prévias ou complicações da gravidez e, mais recentemente, os fatores genéticos (Hackbarthet et al., 2015).

O objetivo deste estudo foi delinear o perfil socioeconômico e obstétrico de mães com filhos pré-termo em um município do semiárido cearense.

\section{Metodologia}

\subsection{Tipo de Estudo}

Trata-se de um estudo descritivo, documental, retrospectivo com abordagem quantitativa. Tal método é essencial para problemas específicos como a prematuridade, uma vez que permite estruturar o aporte teórico desse estudo. Os dados coletados podem ser agrupados em conjuntos e assim, a amostra alcançada pode ser descrita por meio de técnicas de análise matemáticas afim da uniformidade dos resultados (Pereira et al., 2018).

\subsection{Participantes do Local do Estudo}

A população do estudo foram 278 puérperas, correspondendo às mães que tiveram filhos entre agosto e dezembro de 2015. Os critérios de exclusão foram: recém-nascidos de mães cuja caderneta da gestante não estava totalmente preenchidas e que as fichas da Estratégia Trevo de Quatro Folhas estavam incompletas.

O Trevo de Quatro Folhas é uma iniciativa da Prefeitura Municipal de Sobral/Ceará, desenvolvida pela Secretaria de Saúde, que atua com enfoque familiar oferecendo apoio social ao público alvo que se encontra em situação de risco clínico e/ou vulnerabilidade social residentes na área urbana e rural. Tem como objetivo garantir apoio social às gestantes, puérperas e crianças menores de dois anos em risco clínico e/ou vulnerabilidade social e reduzir a mortalidade materno e infantil no município (Albuquerque, 2016).

\subsection{Coleta de Dados}

Utilizou-se como fonte para a coleta, o banco de dados do município em estudo, que é alimentado a partir de entrevistas aplicadas a todas as puérperas que pariram nas maternidades locais, como forma de avaliar a utilização das cadernetas das gestantes. Empregaram-se alguns tópicos como complemento por meio de informações ofertadas pela Estratégia Trevo de Quatro Folhas referentes à: Identificação e características socioeconômicas; História reprodutiva materna e Características da mãe na gravidez. 


\subsection{Procedimentos de análise dos dados}

As informações foram processadas e tabuladas por meio do aplicativo software da área da saúde de epidemiologia EPI INFO 6.0 e do programa computacional Excel 2007 para análise estatística simples.

\subsection{Procedimentos Éticos}

Respeitaram-se os princípios bioéticos preconizados pela Resolução de n $466 / 2012$, do Conselho Nacional de Saúde com o nº do protocolo de aprovação do CEP 56541816.2.0000.5053 e número de parecer 1.590.501.

\section{Resultados}

Verificou-se do perfil socioeconômico no ano de 2015 que 74,6\% das puérperas eram de faixa etária de 20 a 35 anos e 45,7\% possuíam renda inferior a um salário-mínimo. No que tange a escolaridade, o ensino médio completo foi o mais evidenciado em proporção de $37,8 \%$.

Tabela 1: Perfil socioeconômico de mães de recém-nascidos pré-termo no ano de 2015, CEARÁ-Brasil, 2016.

\begin{tabular}{|c|c|c|}
\hline Variáveis & $\mathbf{N}$ & $\%$ \\
\hline \multicolumn{3}{|l|}{ Idade } \\
\hline Até 19 anos & 48 & 17,3 \\
\hline 20 a 35 anos & 207 & 74,6 \\
\hline$>35$ anos & 23 & 8,4 \\
\hline \multicolumn{3}{|l|}{ Renda Familiar } \\
\hline$<1$ salário-mínimo & 127 & 45,7 \\
\hline 1-2 salários-mínimos & 73 & 26,3 \\
\hline 2 ou $<$ salários-mínimos & 63 & 22,7 \\
\hline Desempregada & 15 & 5,4 \\
\hline \multicolumn{3}{|l|}{ Escolaridade } \\
\hline Analfabeta & 01 & 0.4 \\
\hline Alfabetizada & 01 & 0,4 \\
\hline Ens. Fundamental incompleto & 49 & 17,6 \\
\hline Ens. Fundamental completo & 21 & 7,6 \\
\hline Ens. Médio incompleto & 46 & 16,5 \\
\hline Ens. Médio completo & 105 & 37,8 \\
\hline Ens. Superior incompleto & 20 & 7,2 \\
\hline Ens. Superior completo & 31 & 11,2 \\
\hline Pós-graduação & 04 & 1,4 \\
\hline Total & 278 & 100 \\
\hline
\end{tabular}

Fonte: Banco de dados do município (2015).

Quanto ao perfil obstétrico (Tabela 2) 88,1\% apresentaram 01 a 03 gestações e 51,1\% realizaram 07 ou mais consultas de pré-natal. O tipo de parto mais prevalente foi o cesáreo com $69,8 \%$. 
Research, Society and Development, v. 10, n. 3, e2710312769, 2021

(CC BY 4.0) | ISSN 2525-3409 | DOI: http://dx.doi.org/10.33448/rsd-v10i3.12769

Tabela 2: Perfil obstétrico de mães de recém nascidos pré-termo no ano de 2015, CEARÁ-Brasil, 2016.

\begin{tabular}{|c|c|c|}
\hline Variáveis & $\mathbf{N}$ & $\%$ \\
\hline \multicolumn{3}{|c|}{ Número de gestações } \\
\hline 01 a 03 & 245 & 88,1 \\
\hline 04 a 07 & 32 & 11,6 \\
\hline 08 ou $>$ & 01 & 0,4 \\
\hline \multicolumn{3}{|c|}{ Número de consultas } \\
\hline 01 a 03 & 14 & 5,1 \\
\hline 04 a 06 & 69 & 24,9 \\
\hline$\geq 07$ & 142 & 51,1 \\
\hline Sem informação & 53 & 19,1 \\
\hline \multicolumn{3}{|l|}{ Tipo de parto } \\
\hline Cesárea & 194 & 69,8 \\
\hline Normal & 84 & 30,2 \\
\hline Total & 278 & 100 \\
\hline
\end{tabular}

Fonte: Banco de dados do município (2015).

Evidenciou-se que a infecção urinária 90 (32,37\%), a ameaça de trabalho de parto prematuro 66 (23,74\%) e infecção vaginal $62(22,30)$ ficaram entre as intercorrências maternas mais frequentes associadas ao parto prematuro (Figura 1).

Figura 1: Principais intercorrências de mães de recém-nascidos pré-termo no ano de 2015, Ceará-Brasil, 2016.

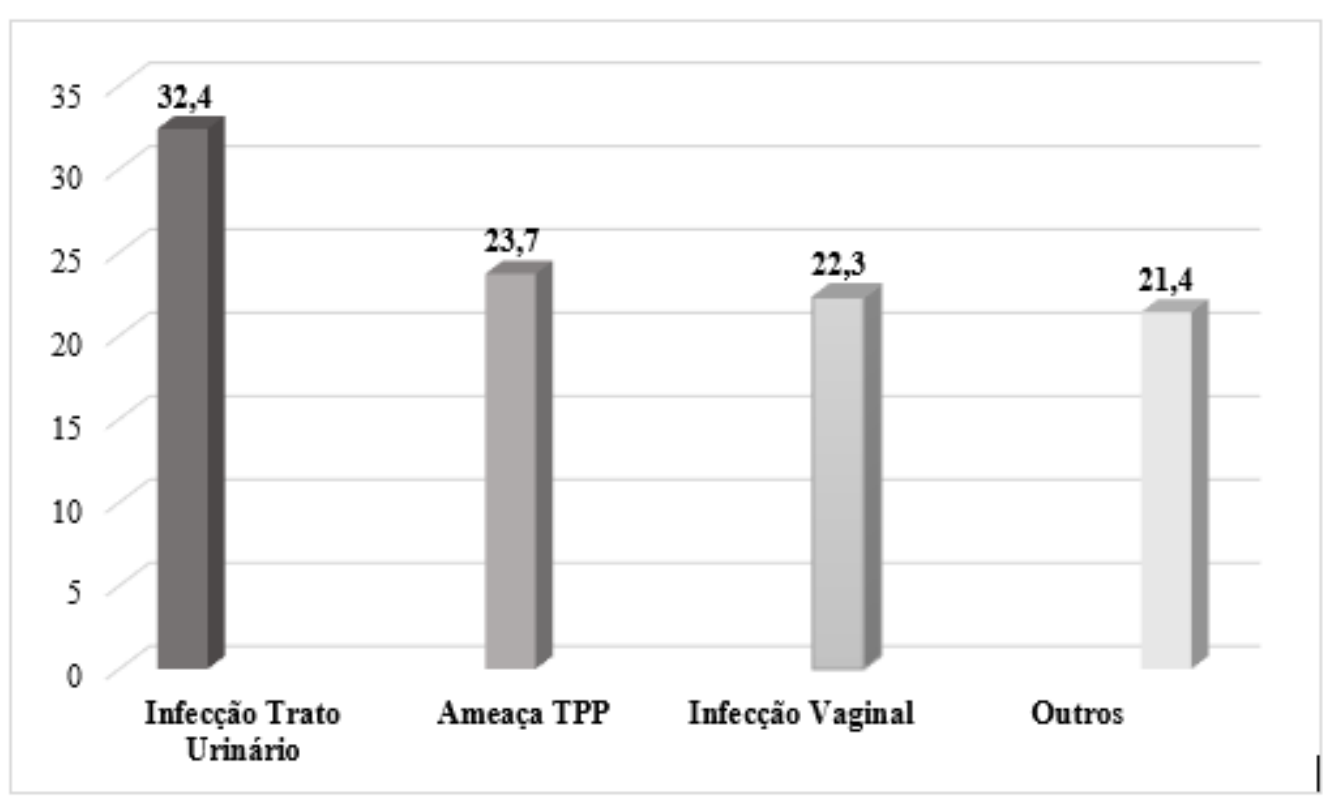

Fonte: Banco de dados do município (2015).

Ao associar intercorrências na gestação com a renda familiar aquelas com menor renda apresentaram maiores percentuais de infecção do trato urinário e infecção do vaginal (Tabela 3). 
Tabela 3: Distribuição percentual do número de puérperas que tiveram Infecção do Trato Urinário/ Infecção Vaginal na gestação de acordo com a Renda Familiar no ano de 2015, Ceará-Brasil, 2016.

\begin{tabular}{c|c|c|c|c|c|c|c|c}
\hline Variável & \multicolumn{5}{|c|}{ Infecção do Trato Urinário } & \multicolumn{3}{c}{ Infecção Vaginal } \\
\hline Renda familiar & SIM & $\%$ & NÃO & $\%$ & SIM & $\%$ & NÃO & \% \\
\hline <1/2 SM & 112 & 4,3 & 20 & 7,1 & 55 & 11,8 & 27 & 99,7 \\
\hline $1 / 2$ SM - 1 SM & 335 & 12,5 & 60 & 21,5 & 29 & 110,4 & 66 & 223,7 \\
\hline 1 - 2 SM & 222 & 8 & 51 & 18,3 & 117 & 66,1 & 56 & 220,1 \\
\hline 2 - 3 SM & 99 & 3,2 & 29 & 10,4 & 55 & 11,8 & 33 & 111,8 \\
\hline $3-5$ SM & 55 & 2 & 16 & 6 & 22 & 11 & 19 & 66,8 \\
\hline > 6 SM & 11 & 0,3 & 3 & 1,1 & 11 & 00,3 & 3 & 11,1 \\
\hline Desempregada & 66 & 2,1 & 9 & 3,2 & 33 & 11,1 & 12 & 44,3 \\
\hline
\end{tabular}

Fonte: Banco de dados do município (2015).

\section{Discussão}

A prevalência de partos prematuros em mulheres com idade entre 20 a 35 anos (74,6\%) grupo considerado de menor risco obstétrico pelo Ministério da Saúde. Entretanto, nesse estudo, essa associação não foi evidenciada. Alguns autores relacionam a prematuridade com extremos da vida reprodutiva < 20 e > 40 anos (Balbi et al., 2016). Outros estudos verificaram que os extremos de idade das mães influenciam os nascimentos de crianças prematuras. A frequência de recémnascidos prematuros, pós termo, de baixo peso, macrossômicos, além de óbitos fetais, é maior em mulheres com idade superior a 35 anos. Já na adolescência, as condições socioeconômicas e geográficas e dificuldades de acesso aos serviços de saúde podem potencializar as intercorrências relativas à gravidez, como a prematuridade (Guimarães et al., 2017). Assim, a variável idade materna está associada a fatores sociodemográficos, porém essa associação não foi evidenciada nesse estudo; contudo, os dados ratificam os achados de outro estudo, em que 76,15\% das mães de partos pré-maturos, correspondiam a faixa etária de 20 a 35 anos (Teixeira et al., 2018).

Identificou-se nesta pesquisa que as mães pertencem às famílias em situação de vulnerabilidade e risco social, havendo predominância $(45,7 \%)$ da renda familiar inferior a um salário-mínimo, corroborando com um estudo realizado na cidade de Patos - PB, em que prevaleceram mães com renda inferior a um salário-mínimo 62,5\%. (Alves et al., 2015).

Esses dados refletem a realidade socioeconômica do município e deve-se também ao fato de que a maior parte das gestantes estudadas foram atendidas em serviços credenciados ou pertencentes ao SUS. Portanto, as características socioeconômicas são determinantes da saúde da díade mãe-bebê, pois os fatores de renda e moradia inapropriadas mantém as mães inerentes a condições alimentícias inadequadas, além de potencializar agravantes do estado de doença por meio da excessiva quantidade de moradores dentro de um mesmo domicílio que em sua maioria apresenta poucos cômodos, corroborando para um elevado grau de infecções por contato direto (Carvalho et al., 2018). 
Quanto à escolaridade das mulheres, notou-se que 37,8\% têm o ensino médio completo, no entanto, esses estudos apresentaram 4 a 7 anos como predomínio (Witt, 2014). Ressalta-se que o baixo nível de escolaridade prejudica a realização de uma assistência pré-natal qualificada e integral, pois as gestantes ao não possuírem conhecimento sobre os benefícios desse cuidado. Assim, apresentam pouca adesão às consultas durante o ciclo gravídico-puerperal, deixando de identificar precocemente prováveis intercorrências e futuras complicações (Santos et al., 2014). Pesquisa realizada no Distrito Federal mostrou que 10,6\% das mães de recém-nascidos prematuros possuía o ensino médio completo (Ferrares, 2016).

Em Bruxelas, uma análise da relação entre os cuidados pré-natais e o risco de parto prematuro também identificou que a escolaridade materna não interferiu na prematuridade. Não obstante, é preciso considerar que a baixa escolaridade da mãe predispõe situações de risco materno e infantil, como a dificuldade de acesso às informações e a medidas de prevenção e promoção da saúde, restrições intelectuais ao exercício da cidadania e da capacidade de autocuidado e busca de assistência (Guimarães et.al., 2017).

No entanto, no Canadá as taxas de prematuridade diminuíam conforme aumentava a escolaridade das mulheres, sendo de 7,9\% nas mulheres com menos de 11 anos de estudo e de 4,9\% em mulheres com mais de 17 anos de estudo (Auger et al., 2013). A prematuridade também estava associada a piores condições de vida, visto que as participantes com escolaridade inadequada apresentavam maior frequência do desfecho referido (Oliveira et.al., 2016).

O número de gestações predominantes foi de uma a três gestações com $(88,1 \%)$. A paridade em associação com fatores sociodemográficos tais como baixa escolaridade e idade, podem elevar o risco de nascimento prematuro (Salge et al., 2017). A gravidez indesejada $(21,10 \%)$ e a gravidez não planejada $(59,63 \%)$ foram evidenciadas indicando a gestações de bebês prematuros ocorrendo em situações de vida desfavoráveis, bem como as fragilidades das políticas de saúde materno e infantil, com destaque para as ações sobre planejamento reprodutivo, que interferem nas várias interfaces do processo de gestação e nascimento (Teixeira et al., 2019).

A quantidade de consultas pré-natal atendeu ao preconizado pelo Ministério da Saúde (Brasil, 2012), corroborando com os resultados de um estudo em uma maternidade pública do estado do Piauí que destacou a que a maioria (72,6\%) realizou o pré-natal de forma satisfatória. (Lopes et al., 2015). A prematuridade apresentou-se de 23,6\% em mulheres com $\leq 6$ consultas e $6,6 \%$ em mulheres $\geq 7$ consultas, apontando para a relevância no que concerne ao número de consultas pré-natal. A análise da razão de chance ajustada indicou maior chance de prematuridade, baixo peso e óbito perinatal nas gestantes com $\leq 6$ consultas (Nascimento et al., 2017). Ressalta-se que as mulheres classificadas com pré-natal inadequado apresentam um aumento de $18 \%$ na chance de nascimento prematuro (Oliveira et.al., 2016).

Outrossim, vale ainda salientar as especificidades relacionadas ao número de consultas em diferentes países. A Holanda segue o mínimo de 06 consultas, número preconizado também no Brasil, entretanto, na Finlândia o mínimo varia em 15 consultas, e na Bélgica são aconselhadas 10 visitas para primíparas e cerca de 7 para multíparas. Não obstante, os dados evidenciam melhores resultados onde se segue o recomendado pelo órgão de saúde pública e a assistência ocorre de forma integral (Beeckman et.al., 2010). A maior adesão e número de consultas pré-natal revelam desfechos mais favoráveis, contudo, o resultado apresentado leva ao questionamento sobre a qualidade da assistência prestada.

O tipo de parto prevalente nesse estudo foi o cesáreo (69,8\%), reforçando os achados de outros estudos, como o de Minas Gerais em que 62,0\% das mães foram submetidas à cesariana (Guimarães et al., 2017). As taxas de parto cirúrgico, que já eram elevadas no começo da década (43,8\%), cresceram significativamente alcançando 53,4\% (Balbi et al., 2016). Vale ressaltar que essa tendência é contrária as políticas de incentivo ao parto vaginal do Sistema Único de Saúde (SUS) brasileiro, diminuindo os partos operatórios, com intuito de aperfeiçoar a recuperação das mães e minimizar os custos. Encontram-se maiores frequências de prematuridade entre as mulheres com gestação múltipla, com pré-natal inadequado, que tiveram partos induzidos e com tipo de parto cesariano. Ainda quanto ao tipo de parto, a cesariana apresentou maior proporção de 
nascimentos prematuros, os recém-nascidos por cesariana tiveram 15\% a mais de chances de serem prematuros (Oliveira et.al., 2016).

Torna-se frequente no período gravídico-puerperal as infecções do trato urinário (ITU), chegando a ser a terceira ocorrência clínica mais comum devido às mudanças anatômicas e fisiológicas do trato urinário, que ocorrem durante esse período, agravando o prognóstico materno (Witt, 2014)

O trabalho de parto prematuro (TPP) é responsável por $75 \%$ dos nascimentos antes da $37^{\mathrm{a}}$ semana de gestação, sendo também responsável por 75 a 95\% dos óbitos neonatais, quando excluídas as malformações congênitas e 50\% das anormalidades neurológicas. Desse modo, sua prevenção poucas vezes é possível no pré-natal devido ter etiologia multifatorial ou desconhecida. As circunstâncias que desencadeiam o TPP podem estar associadas a fatores epidemiológicos, obstétricos e ginecológicos, além dos fatores clínico-cirúrgicos, como as doenças maternas, infecções geniturinárias e procedimentos cirúrgicos na gravidez (Ferreira et al., 2018). Todavia, o Brasil ainda ocupa posição de destaque entre os 10 países com as taxas mais elevadas de prematuridade, mais de $60 \%$ dos nascimentos prematuros ocorrem na África e no sul da Ásia, entretanto, o problema não deixa de ter um viés global fortalecendo a importância das políticas públicas voltadas para esse público, do processo de educação em saúde em todos os níveis de assistência e da adequação aos aspectos específicos e particularidades de cada gestante (Who, 2018).

No que se refere à renda familiar, a maioria das mães pertence ao grupo que possui menos que um salário-mínimo vigente no país, como identificado na tabela 3 , em que a infecção urinária e a vaginal estão diretamente relacionadas ao trabalho de parto prematuro. A infecção do trato urinário mostrou-se altamente associada ao corrimento vaginal. A estratégia do rastreamento rotineiro da bacteriúria assintomática, apesar de eficaz, encontra fortes obstáculos, principalmente em países em desenvolvimento (Veiga, 2017).

A infecção genital destaca-se diante dos diversos processos fisiopatológicos envolvidos no parto pré-termo. Microrganismos presentes na flora vaginal podem atravessar as membranas ovulares, mesmo íntegras, em direção à cavidade amniótica e à decídua, onde são produzidos mediadores inflamatórios responsáveis pelo esvaecimento cervical e contrações uterinas, entretanto, apenas uma pequena parcela de casos de infecção genital é acompanhada por infecção uterina e nascimento prematuro (Paganoti et al., 2015).

Desse modo, devido à prematuridade ter etiologia multifatorial é necessário buscar estratégias que possibilitem o rastreamento de mulheres de maior risco para o parto prematuro. Enfatiza-se também a importância do acompanhamento prénatal para o manejo frente aos possíveis agravos.

\section{Conclusão}

O perfil socioeconômico das mulheres aponta para uma situação de vulnerabilidade econômica que pode influenciar a ocorrência das elevadas taxas de infecções urinárias e vaginais, tendo em vista que as condições financeiras para uso das medicações ou deslocamento à unidade de saúde para busca da medicação tornam-se prejudicadas. Consonante à saúde, há elevado número de consultas de pré-natal, entretanto questiona-se sobre a qualidade da assistência prestada nestas consultas.

Atenta-se ao registro do aumento de partos cesáreos, o que eleva os casos de prematuridade e pode estar estritamente referente a erros na definição da idade gestacional e à ocorrência de cesariana eletiva, aquelas marcadas com antecedência por intervenção médica e por solicitação da mãe.

Salienta-se as limitações deste estudo no que se refere ao preenchimento inadequado as cadernetas de pré-natal que não puderam ser avaliados, devido incompletude dos dados, reduzindo a oportunidade de maiores amostras e portanto 
impermite novos resultados para a pesquisa, privando o refinamento e completude do estudo. Espera-se que a partir desse estudo novas pesquisas investiguem as vulnerabilidades e suas consequências no trabalho de parto prematuro.

Mediante tais conclusões frente à prematuridade em um município cearense espera-se que novas pesquisas sejam oportunizadas. Urge-se estudos que investiguem as vulnerabilidades diante do trabalho de parto prematuro e suas consequências em todo o estado do Ceará. Além disso, fomenta-se a necessidade de novas vertentes do estudo em âmbito regional, estadual e nacional visando possíveis comparações acerca dos diversos cenários em que a prematuridade ocorre afim de evidenciar as causas de sua frequência alarmante.

Instigar novas pesquisas nessa temática em diferentes lugares do Brasil permite uma visão ampliada sobre as facetas da prematuridade e assim, permite suporte teórico para que profissionais e acadêmicos na área da saúde possam comparar e elaborar intervenções eficazes para coarctação de maiores casos de vulnerabilidades, em vista da promoção de um cuidado integral às mães e recém-nascidos prematuros.

\section{Referências}

Albuquerque, I. M. N. (2016). O compromisso com as políticas públicas de saúde: experiências exitosas em Sobral-CE. Sanare Revista de Políticas Públicas. 10(1): 06-07. https://sanare.emnuvens.com.br/sanare/article/view/922/551.

Auger, N., Abrahamowicz, M., Park, A. L., \& Wynant, W. (2013). Extreme maternal education and preterm birth: time-to-event analysis of age and nativitydependent risks. Ann Epidemioly, 23(1):01-06. https://doi.org/10.1016/j.annepidem.2012.10.007

Balbi, B., Leite, M. A. B., \& Parada, C. G. L. (2016). Tendência temporal do nascimento pré-termo e de seus determinantes em uma década. Ciência \& Saúde Coletiva. 21 (1). https://doi.org/10.1590/1413-81232015211.20512015.

Beeckman, K., Louckx, F., \& Putman, K. (2010). Determinants of the number of antenatal visits in a metropolitan region. BMC Public Health. https://doi.org/10.1186/1471-2458-10-527.

Brasil, Ministério da Saúde. (2012). Atenção ao pré-natal de baixo risco. http://bvsms.saude.gov.br/bvs/publicacoes/cadernos_atenc ao_basica_32_prenatal.pdf

Brasil, Ministério da Saúde. (2013). Uma análise da situação de saúde e das doenças transmissíveis relacionadas à pobreza. http://bvsms.saude.gov.br/bvs/publicacoes/saude_brasil_2013_analise_situacao_saude.pdf

Carvalho, J. B. L., Teixeira, G. A., Morais, P. C., Sena, A. V., \& Alves, T. R. M. (2018). Condições socioeconômicas da gestação de bebês prematuros. Revista enfermagem UFPE on line, 12 (2): 386-90. https://doi.org/10.5205/1981-8963-v12i2a15294p386-390-2018.

Ceará - Governo do Estado do Ceará. (2017). Linha-Guia Nascer no Ceará Gestante e Criança Menor de 2 Anos. https://www.saude.ce.gov.br/wpcontent/uploads/sites/9/2018/06/linha_guia_nascer_no_ceara_2017.pdf

Ferreira, J. A. R., Albuquerque, R. A. S., Aragão, S. R., \& Rodrigues, M. E. N. G. (2018). Perfil epidemiológico de mães e recém-nascidos prematuros. Revista Enfermagem Contemporânea.7(1):6-12. https://doi.org/10.17267/2317-3378rec.v7i1.1159

Guimarães, E. A. A., Vieira, C. S., Nunes, F. D. D., Januário, G. C., Oliveira, V. C., \& Dibúrcio, J. D. (2017). Prevalência e fatores associados á prematuridade em Divinópolis, Minas Gerais, 2008-2011: análise do Sistema de Informações sobre Nascidos Vivos. Revista Epidemiologia e Serviços de. Saude. 26 (1): 91-98. https://doi.org/10.5123/s1679-49742017000100010

Gonzaga, I. C. A., Santos, S. L. D., Silva, A. R. V., \& Campelo, V. (2016). Atenção pré-natal e fatores de risco associados à prematuridade baixo peso ao nascer em capital do nordeste brasileiro. Revista Ciência \& Saúde Coletiva. 21 (6). https://doi.org/10.1590/1413-81232015216.06162015.

Hackbarth, B. B., Ferreira, J. A., Carstens, H. P., Amaral, A. R., Silva, M. R., Silva, J. C, \& França, P. H. C de. (2015). Preterm birth susceptibility: investigation of behavioral, genetic, medical and sociodemographic factors. Revista Brasileira Ginecologia e Obstetrícia. 37 (8). https://doi.org/10.1590/SO100-720320150005338

Madi, J. M., Araújo, B. F., Zatti, H., Rombaldi, R. L., Lorencetti, J., Pinson, G. Tonezer, J., \& Caron, F. (2016). Avaliação dos fatores associados à ocorrência de prematuridade em um hospital terciário de ensino. Revista da AMRIGS. 56 (2). https://docplayer.com.br/29411891-Avaliacao-dos-fatores-associados-aocorrencia-de-prematuridade-em-um-hospital-terciario-de-ensino.html

Oliveira, L. L., Gonçalves, A. C., Costa, J. S. D., \& Bonilha, A. L. L. (2016). Maternal and neonatal factors related to prematurity. Revista Escola de Enfermagem. 50(3):382-389. https://doi.org/10.1590/S0080-623420160000400002 
Research, Society and Development, v. 10, n. 3, e2710312769, 2021 (CC BY 4.0) | ISSN 2525-3409 | DOI: http://dx.doi.org/10.33448/rsd-v10i3.12769

Paganoti, C. F., Bittar, R. E., Carvalho, M. H. B., Francisco, R. P. V., \& Zugaib, M. (2015). As infecções genitais podem alterar os resultados dos testes preditivos do parto prematuro? Revista Brasileira de Ginecologia e Obstetrícia. 37(1). https://doi.org/10.1590/SO100-720320140005202

Pereira, A. S., Shitsuka, D. M., Parreura, F.J., \& Shitsuka, R. (2018). Metodologia da pesquisa científica. https://repositorio.ufsm.br/bitstream/há ndle/1/15824/Lic_Computacao_Metodologia-Pesquisa-Cientifica.pdf?sequence=1.

Salge, A. K. M., Vieira, A. V. C., Aguiar, A. K. A., Lobo, S. F., Xavier, R. M., Zatta, L. T., Correa, R. R. M., \& Silva, R. C. R e. (2017). Fatores maternos e neonatais associados à prematuridade. Revista Eletrônica de Enfermagem.11(3). https://www.revistas.ufg.br/fen/article/view/47198/23139.

Santos, N. L., Costa, M. C., Amaral, M. T., Vieira, G. O., Bacelar, E. B., \& Almeida, A. H. (2014). Gravidez na adolescência: análise de fatores de risco para baixo peso, prematuridade e cesariana. Revista Ciência Saúde Coletiva.19 (3). https://doi.org/10.1590/1413-81232014193.18352013

Teixeira, G. A., Carvalho, J. B. L. D., Rocha, B. G. D., Pereira, S. A., \& Enders, B. C. (2018). Perfil de mães e o desfecho do nascimento prematuro ou a termo. Revista Cogitare Enfermagem. 23 (1). https://doi.org/10.5380/ce.v23i1.51409.

Teixeira, G. A., Carvalho, J. B. L. D., Sena, A. V. D., Morais, P. C. D., \& Alves, T. R. D.M. (2019). Características maternas de partos prematuros. Revista Enfermagem Atual In Derme. 81(19). https://revistaenfermagematual.com.br/index.php/revista/article/view/317

Veiga, S. P., Boeira, V. L., Silva, C. M., \& Peder, L. D. (2017). Incidência de infecções do trato urinário em gestantes e correlação com o tempo de duração da gestação. Revista Acta Biomedica Brasiliensia. 8(1). https://doi.org/10.18.571/acbm. 125

Witt, W. P., Cheng. E. R., Wisk, L.E., Litzelman. K., Chatterjee, D., Mandell, K., \& Wakeel, F. (2014). Pretermbirth in the United States: the impact of stress ful life events prior to conceptionand maternal age. American Journal Public Health. 104 (73). https://doi.org/10.2105 / AJPH.2013.301688

Who, World Health Organization. (2018). Preterm birth. http://www.who.int/mediacentre/factsheets/fs363/en/ 